\title{
The SGK3-triggered ubiquitin-proteasome degradation of podocalyxin (PC) and ezrin in podocytes was associated with the stability of the PC/ezrin complex
}

\author{
Ya-Pei Yuan ${ }^{1}$, Hong Zhao ${ }^{2}$, Li-Qin Peng ${ }^{1,3}$, Zi-Fang Li', Song Liu', Cheng-Yan Yuan' ${ }^{1}$, Mercy-Julian Mwamunyi ${ }^{1}$, \\ David Pearce ${ }^{4,5}$ and Li-Jun Yao ${ }^{1}$
}

\begin{abstract}
Podocyte damage is commonly accompanied by destabilization of the podocalyxin (PC)/ezrin complex. Serum- and glucocorticoid-inducible kinase 3 (SGK3) plays a role in the maintenance of podocyte function, but the details of this role are poorly understood. Herein we demonstrated that SGK3 and its downstream target protein neural precursor cell expressed developmentally downregulated protein 4 subtype 2 (Nedd4-2) triggered PC and ezrin interaction. In adriamycin (ADR)-induced nephritic mice, and after puromycin aminonucleoside (PAN)-induced podocyte damage in vitro, PC and ezrin protein expression levels decreased significantly, while Nedd4-2 activity increased. Moreover, PAN treatment increased PC and ezrin ubiquitination and decreased PC/ezrin interaction in cultured mouse podocytes. The downregulation of SGK3 activity in mouse podocytes resulted in decreased PC and ezrin protein expression and increased the ubiquitin-proteasome degradation of PC and ezrin. Furthermore, upregulation of SGK3 activity mostly reversed the PAN-induced decrease in PC and ezrin protein expression. Overexpression of Nedd4-2 led to decreased ezrin protein expression via the upregulation of ezrin ubiquitination. In contrast, Nedd4-2 knockdown resulted in increased ezrin protein expression but decreased ezrin ubiquitination. In PC-transfected human embryonic kidney (HEK293T) cells, SGK3 activity downregulation and Nedd4-2 overexpression resulted in decreased PC/ezrin interaction. These results suggested that SGK3 triggers the ubiquitin-proteasome degradation of PC and ezrin, while the SGK3/ Nedd4-2 signaling pathway regulates ezrin, but not PC, ubiquitination. Thus SGK3 helps to regulate podocyte function by maintaining the stability of the PC/ezrin complex.
\end{abstract}

\section{Introduction}

Proteinuria, an important clinical manifestation of chronic kidney disease (CKD), may be caused by the loss of podocyte structural protein function ${ }^{1-3}$, leading to glomerular sclerosis and end-stage renal failure. In order

\footnotetext{
Correspondence: L.-J. Yao (drylj@hotmail.com)

${ }^{1}$ Department of Nephrology, Union Hospital, Tongji Medical College, Huazhong University of Science and Technology, 430022 Wuhan, China ${ }^{2}$ Department of Trauma Surgery, Tongji Hospital, Tongji Medical College, Huazhong University of Science and Technology, 430030 Wuhan, China Full list of author information is available at the end of the article. These authors contributed equally: Ya-Pei Yuan, Hong Zhao.
} Edited by A. Linkermann to understand pathogenesis of proteinuria and to design therapeutic strategies to prevent the development of proteinuric CKD, investigations of the signaling pathways underlying podocyte damage are necessary.

We previously reported that a lack of serum- and glucocorticoid-inducible kinase 3 (SGK3) resulted in proteinuria in mice and that SGK3 activity and expression were inhibited after podocyte damage, both in vivo and in vitro ${ }^{4}$. Other studies have shown that the downstream target protein of SGK3, glycogen synthase kinase-3 (GSK3), is involved in the regulation of podocytosis, proteinuria, and podocyte autonomic injury ${ }^{5,6}$. Like AKT (also known as protein kinase

\section{(c) The Author(s) 2018}

(c) (i) Open Access This article is licensed under a Creative Commons Attribution 4.0 International License, which permits use, sharing, adaptation, distribution and reproduction cc in any medium or format, as long as you give appropriate credit to the original author(s) and the source, provide a link to the Creative Commons license, and indicate if changes were made. The images or other third party material in this article are included in the article's Creative Commons license, unless indicated otherwise in a credit line to the material. If material is not included in the article's Creative Commons license and your intended use is not permitted by statutory regulation or exceeds the permitted use, you will need to obtain permission directly from the copyright holder. To view a copy of this license, visit http://creativecommons.org/licenses/by/4.0/. 
B), SGK3 is a member of the serine-threonine protein kinase family and is highly expressed in mammalian kidney tissue $^{7}$. Extensive studies have shown that AKT2 regulates the survival and function of podocytes during $\mathrm{CKD}^{8-10}$. AKT2 and SGK3 are fairly homologous in structure and function $^{11}$. Thus it is probable that SGK3 is involved in the pathogenesis of proteinuria after podocyte damage ${ }^{4}$. However, the mechanism by which SGK3 induces podocyte dysfunction remains poorly understood.

Podocalyxin (PC) is a heavily sialylated and sulfated membrane protein expressed on the apical surface of glomerular epithelial cells (podocytes) ${ }^{12,13}$. PC is composed of a mucin domain, a disulfide-bonded globular domain, a transmembrane region, and a highly charged cytoplasmic tail that has potential phosphorylation sites for protein kinase $\mathrm{C}$ and casein kinase $\mathrm{II}^{14}$. This highly conserved cytoplasmic PC tail interacts with cytoskeletal actin through the $\mathrm{Na}^{+} / \mathrm{H}^{+}$exchange Factor 2 (NHERF2)/ezrin complex to maintain podocyte morphology, motility, and antiadhesion $^{15-18}$. A number of studies have shown that urinary PC can be used as a marker for podocyte damage ${ }^{19,20}$. Ezrin, a member of the ezrin-radixin-moesin (ERM) protein family, maintains the stability of $\mathrm{PC}$ on the podocyte membrane ${ }^{21,22}$. The deletion of the PC/ezrin complex results in the shedding of $\mathrm{PC}$ from the podocyte membrane, the disappearance of the charged filtration barrier from the membrane, the fusing of the podocyte foot processes, and proteinuria ${ }^{17}$. Although it has been reported that the dissociation of the PC/ezrin complex leads to the disruption of foot process architecture ${ }^{23,24}$, the details of the molecular mechanisms connecting PC/ezrin complex disruption to podocyte damage remain unclear.

The neural precursor cell expressed developmentally downregulated protein 4 subtype 2 (Nedd4-2) protein is a downstream target of SGK3 ${ }^{7,25}$. Nedd4-2 is a member of the homologous to E6-AP C-terminus (HECT) ubiquitin (Ub) ligase E3 family and is mainly distributed in the mammalian livers, kidneys, lungs, hearts, and brains. Nedd4-2 binds to the PPxY (PY) motif of its target proteins, recognizing and labeling target proteins with $\mathrm{Ub}$ to initiate ubiquitin-mediated protein degradation ${ }^{26,27}$. Unlike PC, ezrin has a PY motif that may bind the WW domain of Nedd4-2. However, it is unknown whether Nedd4-2 regulates the expression and function of ezrin in podocytes by mediating ezrin ubiquitination. Thus we here investigated the effects of SGK3 and the SGK3/ Nedd4-2 signaling pathway on PC and ezrin ubiquitination, as well as on PC/ezrin interaction.

\section{Results}

PC and ezrin were downregulated in the adriamycin (ADR) mouse model

As previous studies have shown that ezrin downregulation or the inhibition of $\mathrm{PC} /$ ezrin complex activity can lead to podocyte dysfunction ${ }^{28-30}$, we first investigated whether the expression levels of PC and ezrin were altered after podocyte damage in an ADRinduced nephritic mouse model, which was established as previously described ${ }^{4}$. By 14 days after ADR injection, ADR-treated mice had developed significant proteinuria in comparison with the control group (Fig. 1a). Morphological kidney injuries were clearly shown by periodic acid-Schiff (PAS) staining 2 weeks after ADR injection. These injuries were characterized by glomerular sclerotic lesions and by a tubular dilation with an expanded lumen loaded with proteins (Fig. 1b). Both PC and ezrin were significantly downregulated in the kidneys of ADR mice, as compared to the control mice (Fig. 1c). PC was highly expressed in the glomerulus and peritubular capillaries of the mice, while ezrin was expressed in the glomerulus and the renal tubules. Ezrin was also known as a marker of activated or damaged podocytes in experimental models ${ }^{28}$. PC and ezrin expression levels, as shown by immunohistochemical staining, were significantly lower in the glomeruli of ADR mice than in the glomeruli of control mice (Fig. 1d). Thus, consistent with previous studies ${ }^{30,31}$, PC and ezrin were downregulated after podocyte damage in vivo.

\section{Puromycin aminonucleoside (PAN) treatment disrupted the PC/ezrin interaction in cultured mouse podocytes by inhibiting $\mathrm{PC}$ and ezrin protein expression}

To explore PC and ezrin expression after podocyte damage in vitro, mouse podocytes were cultured and treated with $50 \mu \mathrm{g} / \mathrm{ml}$ PAN for $24 \mathrm{~h}$. As demonstrated by 3-(4, 5-dimethyl-2-thiazolyl)-2,5-diphenyl-2-H-tetrazolium bromide (MTT) assays and western blots, PAN treatment reduced podocyte viability and increased the expression of the podocyte damage maker desmin in comparison with control cells (Fig. 2a, b). This indicated that an in vitro PAN-induced podocyte damage model had been successfully generated. In addition, F-actin was distributed as parallel bundles of stress fibers in the control podocytes; PAN treatment destroyed these stress fibers (Fig. 2c). Reverse transcriptase (RT)-PCR analysis showed that PC mRNA expression in mouse podocytes increased significantly after PAN treatment, as compared to untreated mouse podocytes, while ezrin mRNA expression was unchanged (Fig. 2d). However, PC and ezrin protein expression levels were dramatically lower in the mouse podocytes treated with PAN than in the control mouse podocytes (Fig. 2e).

Because PC and ezrin are co-expressed in podocytes, we next used confocal microscopy and coimmunoprecipitation (Co-IP) assays to test whether PAN regulated the PC/ezrin interaction. Confocal 

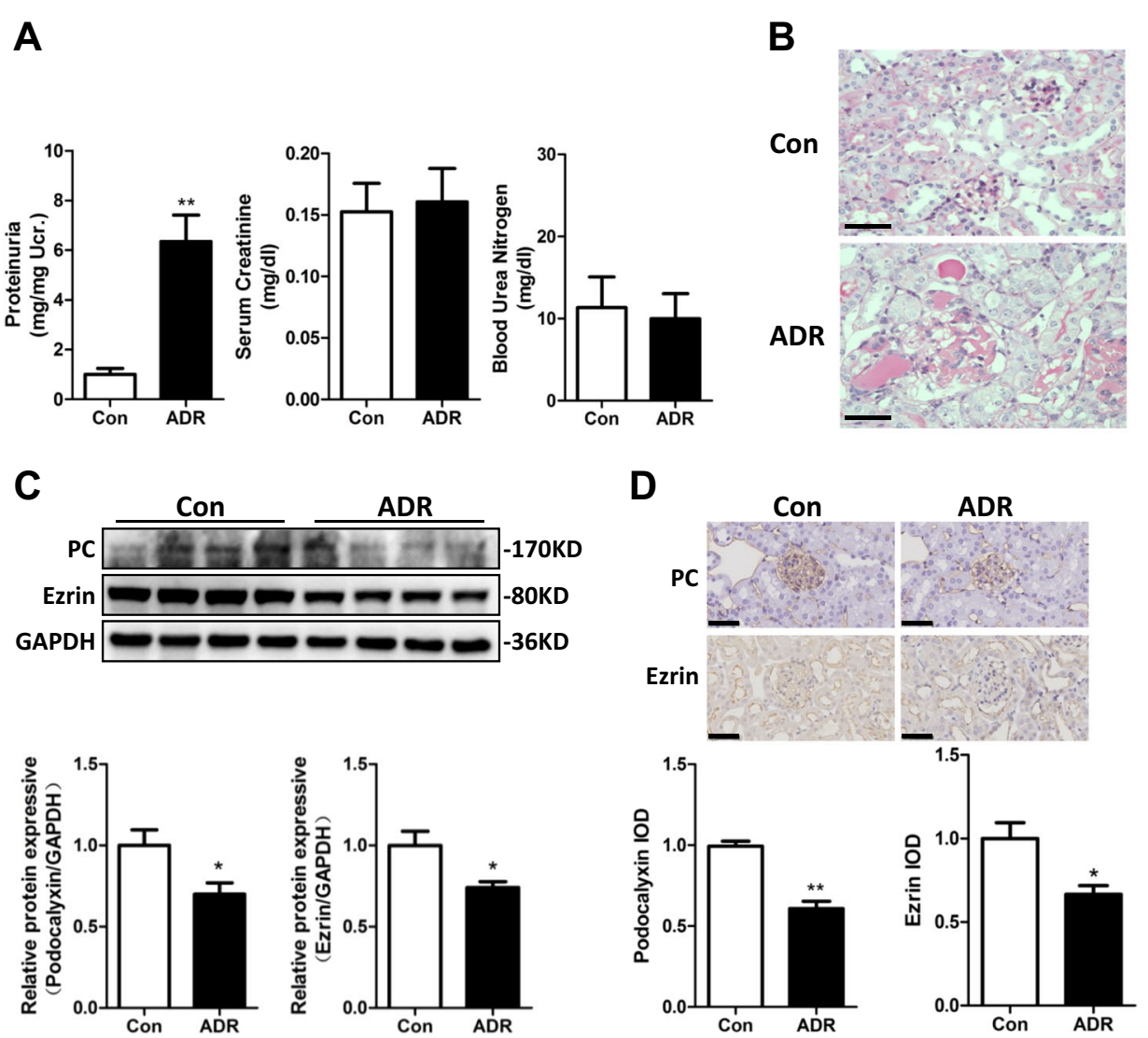

Fig. 1 The expression levels of PC and ezrin in an ADR mouse model. An intravenous injection of ADR (10.5 mg/kg) was used to induce nephritis in the mouse model. a Urinary albumin levels, blood urea nitrogen and serum creatinine in mice at 14 days after ADR injection. Urinary albumin excretion were presented as $\mathrm{mg} / \mathrm{mg}$ of urinary creatinine. $\mathbf{b}$ Representative images of PAS staining micrographs show kidney injury at 2 weeks after ADR injection in different groups of mice. Scale bar $=50 \mu \mathrm{m}$. c Top panel: immunoblot analysis of PC and ezrin expression in control and ADRinjected mice; GAPDH was used for normalization. Bottom panel: densitometric quantification of PC and ezrin. $\mathbf{d}$ Top panel: immunohistochemical staining shows PC and ezrin expression in the glomerulus after ADR injury. Mice were sacrificed 14 days after ADR injection, and paraffin-embedded kidney sections were stained with PC and ezrin antibodies. Bottom panel: computerized morphometric quantification of PC and ezrin staining in the glomeruli of control and ADR mice. Scale bar $=50 \mu \mathrm{m} ; n=6 /$ group; ${ }^{*} P<0.05$ vs. control mice; ${ }^{*} P<0.01$ vs. control mice

immunostaining revealed that PAN treatment decreased interaction between $\mathrm{PC}$ and ezrin in cultured mouse podocytes (Fig. 2f). Co-IP assays indicated that the PC/ezrin interaction in mouse podocytes treated with PAN was reduced as compared to control cells, indicating that PAN was directly involved in the co-precipitation of PC and ezrin (Fig. 2g). Our results therefore suggested that PAN treatment disrupted the stability of the PC/ezrin complex, implying that the PAN-induced decrease in $\mathrm{PC}$ and ezrin protein expression in mouse podocytes might not be due to the effect of PAN on $\mathrm{PC}$ and ezrin mRNA transcription.

PAN increased the ubiquitin-mediated protein degradation of PC and ezrin in cultured mouse podocytes

The decreased protein expression of PC and ezrin induced by PAN might be the result of increased PC and ezrin protein degradation. To test this hypothesis, we administered the proteasome inhibitor MG132 after PAN treatment to reduce ubiquitin-mediated protein degradation. When MG132 was added after PAN treatment, the protein expression levels of $\mathrm{PC}$ and ezrin no longer decreased (Fig. 3a). We next determined the degree of $\mathrm{PC}$ and ezrin ubiquitination after PAN treatment. As no commercial PC antibodies for Co-IP are yet available, we transfected mouse podocytes with a Ub-FLAG plasmid for $48 \mathrm{~h}$ and treated mouse podocytes with PAN for the last $24 \mathrm{~h}$ of transfection. The ubiquitination levels of PC (Fig. 3b) and ezrin (Fig. 3c) increased remarkably in the PAN-treated mouse podocytes as compared to the control mouse podocytes, suggesting that ubiquitin-mediated degradation of the PC and ezrin proteins is involved in PAN-mediated podocyte damage. 

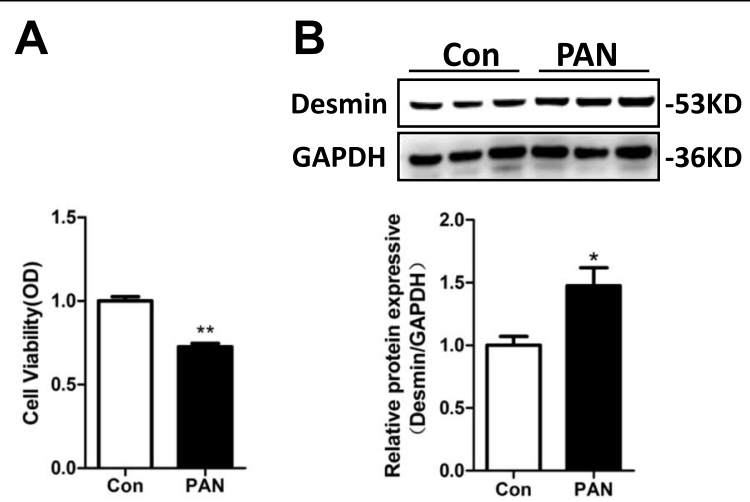

C

D
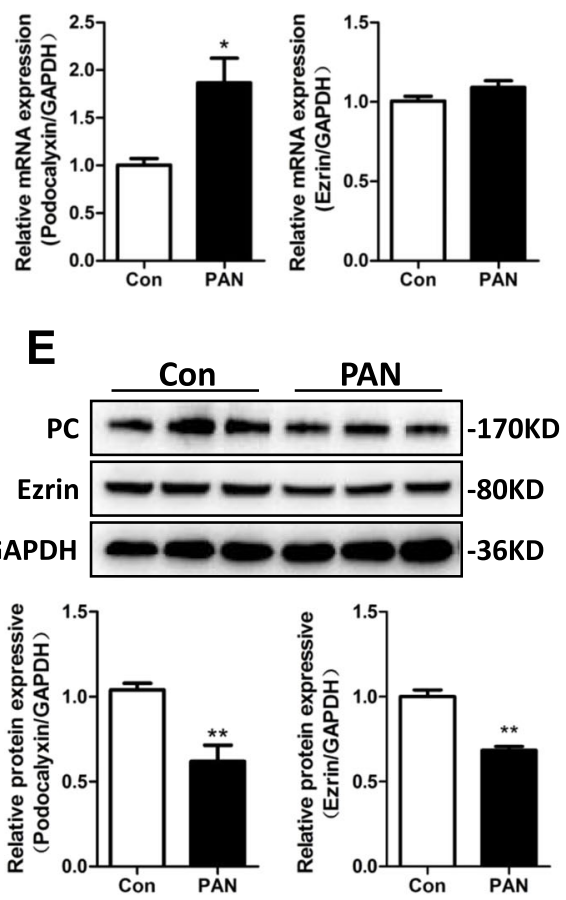

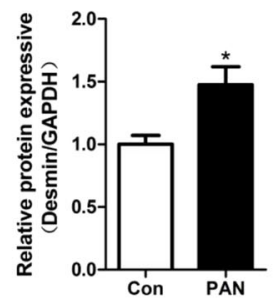

36KD
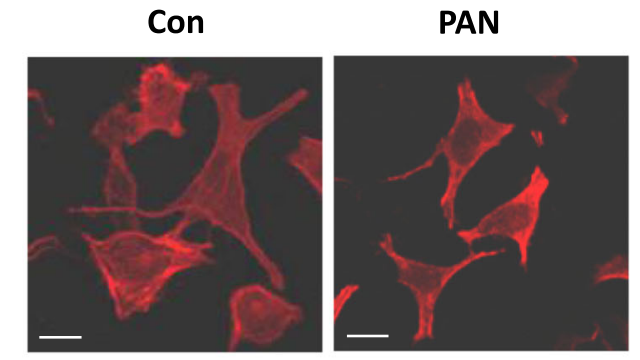

$\mathbf{F}$
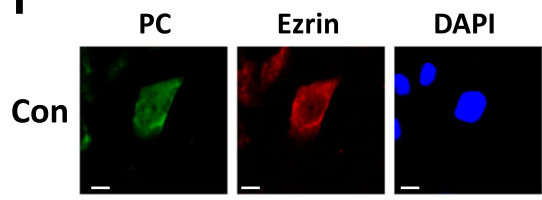

Merge

PAN
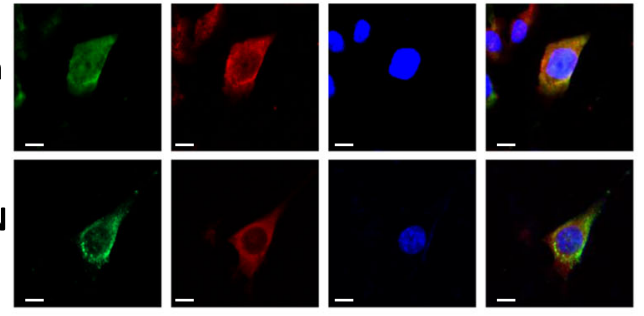

G

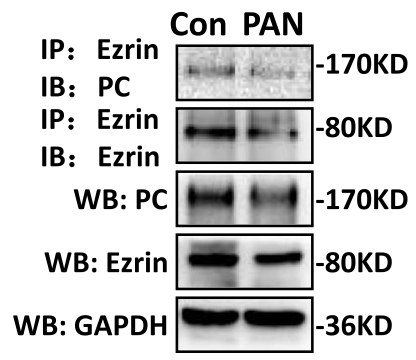

Fig. 2 PAN inhibited PC and ezrin protein expression and downregulated PC/ezrin interaction in cultured mouse podocytes. Immortalized mouse podocytes were exposed to $50 \mu \mathrm{g} / \mathrm{ml}$ PAN for $24 \mathrm{~h}$. a MTT assay was used to measure cell viability after PAN treatment for $24 \mathrm{~h}$. b Top panel: Immunoblot analysis for desmin expression; GAPDH was used for normalization. Bottom panel: Quantification of desmin by densitometry. c Immunofluorescence staining showed F-actin rearrangement in PAN-treated podocytes. Scale bar $=25 \mu \mathrm{m}$. d After PAN treatment, PC and ezrin mRNA expression levels were detected with qRT-PCR. e Upper panel: immunoblot analysis of PC and ezrin protein expression. Bottom panel: densitometric quantification of PC and ezrin. Data are means \pm SEs of three independent experiments. ${ }^{*} P<0.05$ vs. the control group; ${ }^{* *} P<0.01$ vs. the control group. $\mathbf{f}$ Confocal immunofluorescence images show PC and ezrin staining in cultured podocytes after treatment with PAN. Scale bar $=$ 25 mm. g After PAN treatment, whole-cell lysates (WCLs) were precipitated with an anti-ezrin antibody. The immunoprecipitates were analyzed with a western blot using anti-ezrin and anti-PC antibodies. PC and ezrin protein expression in WCLs was analyzed with an immunoblot

SGK3-mediated PC and ezrin protein expression was involved in PAN-mediated podocyte damage

We recently reported that SGK3 was involved in the ADR-induced nephritic mouse model and in the PAN-induced podocyte damage cell model ${ }^{4}$. However, it remains unclear whether SGK3 participates in the regulation of $\mathrm{PC}$ and ezrin protein expression. In cultured mouse podocytes transfected with an eukaryotic expression vector encoding the constitutively inactivated K191M mutant of SGK3 (SGK3-K191M), PC mRNA expression was significantly reduced as compared to control cells, but ezrin mRNA expression was unaffected (Fig. 4a). The protein expression levels of PC and ezrin in the SGK3-K191M-transfected mouse podocytes 


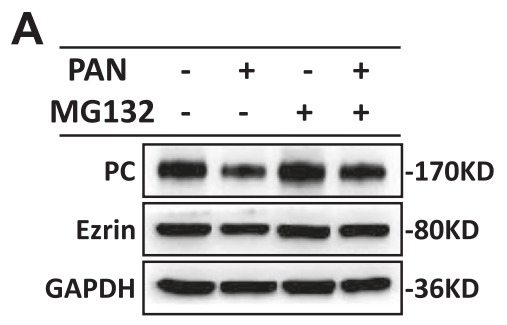

B

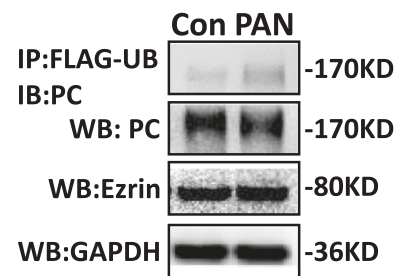

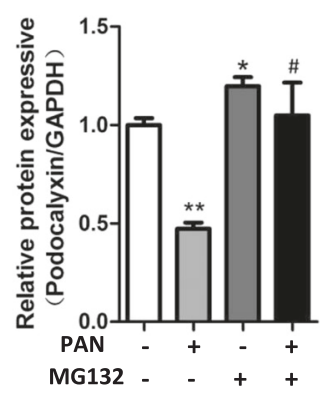

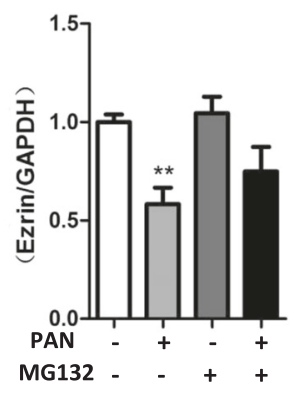

C

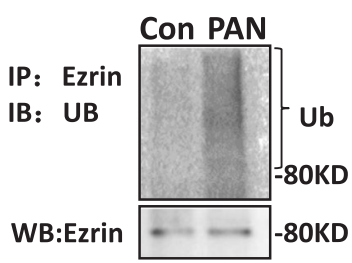

Fig. 3 PAN enhanced the ubiquitin-mediated degradation of PC and ezrin in cultured mouse podocytes. a After treatment with PAN, mouse podocytes were either untreated or treated with $10 \mu \mathrm{M} \mathrm{MG132}$ for the last $8 \mathrm{~h}$ of transfection. Left panel: immunoblot analysis showing PC and ezrin expression. GAPDH was used for normalization. Right panel: densitometric quantification of PC and ezrin. Data are means \pm SEs of three independent experiments. ${ }^{*} P<0.05$ vs. the PAN ${ }^{-} / \mathrm{MG} 132^{-}$cells, ${ }^{* *} P<0.01$ vs. the PAN ${ }^{-} / \mathrm{MG} 132^{-}$cells. ${ }^{\#} P<0.05$ vs. the PAN ${ }^{+} / \mathrm{MG} 132^{-}$cells. $\mathbf{b}$ Mouse podocytes were transfected with a Ub-FLAG plasmid for $48 \mathrm{~h}$ and treated with PAN for the last $24 \mathrm{~h}$ of transfection. The proteasome inhibitor MG132 was added to the mouse podocytes for the last $8 \mathrm{~h}$ of transfection to prevent protein degradation. WCLs were precipitated with anti-FLAG antibodies, and the immunoprecipitates were immunoblotted with anti-PC antibodies. PC and ezrin expression was measured in WCLs after PAN treatment using immunoblotting. c Mouse podocytes were treated with PAN for $24 \mathrm{~h}$ and with MG132 for the last $8 \mathrm{~h}$ of PAN treatment. WCLs were precipitated with anti-ezrin antibodies, and the immunoprecipitates were immunoblotted with anti-ezrin and anti-ubiquitin antibodies

were significantly lower than the $\mathrm{PC}$ and ezrin expression levels in the scramble plasmid-transfected mouse podocytes (Fig. 4b). This suggested that SGK3 might be involved in the regulation of $\mathrm{PC}$ and ezrin expression.

To further investigate the involvement of SGK3 in the downregulation of PC and ezrin after PAN-mediated podocyte injury, cultured mouse podocytes were transfected with the constitutively activated S486D mutant of SGK3 (SGK3-S486D) or scramble plasmid for $48 \mathrm{~h}$ and treated with PAN for the last $24 \mathrm{~h}$ of transfection. We found that PC and ezrin expression levels were significantly lower in the $\mathrm{PAN}^{+} / \mathrm{S}_{486 \mathrm{D}^{-}}$transfected mouse podocytes, as compared to the $\mathrm{PAN}^{-} / \mathrm{S}_{486 \mathrm{D}^{-}}$-transfected mouse podocytes. Notably, the expression levels of PC and ezrin in the $\mathrm{PAN}^{+} / \mathrm{S}_{486 \mathrm{D}^{+}}$-transfected mouse podocytes were almost equivalent to those in the $\mathrm{PAN}^{-}$/

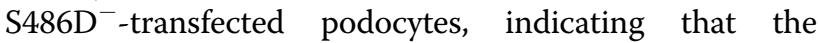
increased activation of SGK3 mostly reversed the PANinduced downregulation of $\mathrm{PC}$ and ezrin. In addition, PC expression did not differ significantly between the $\mathrm{PAN}^{+} / \mathrm{S}_{486 \mathrm{D}}{ }^{+}$- and the $\mathrm{PAN}^{-} / \mathrm{S}_{486 \mathrm{D}^{+} \text {-transfected }}$ mouse podocytes, suggesting that PAN treatment did not further downregulate $\mathrm{PC}$ protein expression in mouse podocytes transfected with constitutively activated SGK3 (Fig. 4c).

To study the role of SGK3 in the PC/ezrin complex, mouse podocytes were transfected with either the SGK3-K191M or the SGK3-S486D plasmid in vitro. PC and ezrin binding was then measured with Co-IP assays. We found that, compared with untransfected mouse podocytes, the interaction between $\mathrm{PC}$ and ezrin decreased in mouse podocytes transfected with SGK3K191M but increased significantly in mouse podocytes transfected with SGK3-S486D (Fig. 4d). Therefore, ourresults indicated that SGK3 not only alleviated the PAN-induced downregulation of PC and ezrin but also maintained PC/ezrin complex stability.

\section{SGK3 triggered ubiquitin-mediated degradation of PC and ezrin in cultured mouse podocytes}

To explore how SGK3 affected the protein expression of PC and ezrin, the SGK3-K191M plasmid was transfected into mouse podocytes to decrease SGK3 activity. The proteasome inhibitor MG132 was added for the last $8 \mathrm{~h}$ of transfection to inhibit protein degradation. The downregulation of PC and ezrin in SGK3-K191Mtransfected mouse podocytes was mostly reversed by 

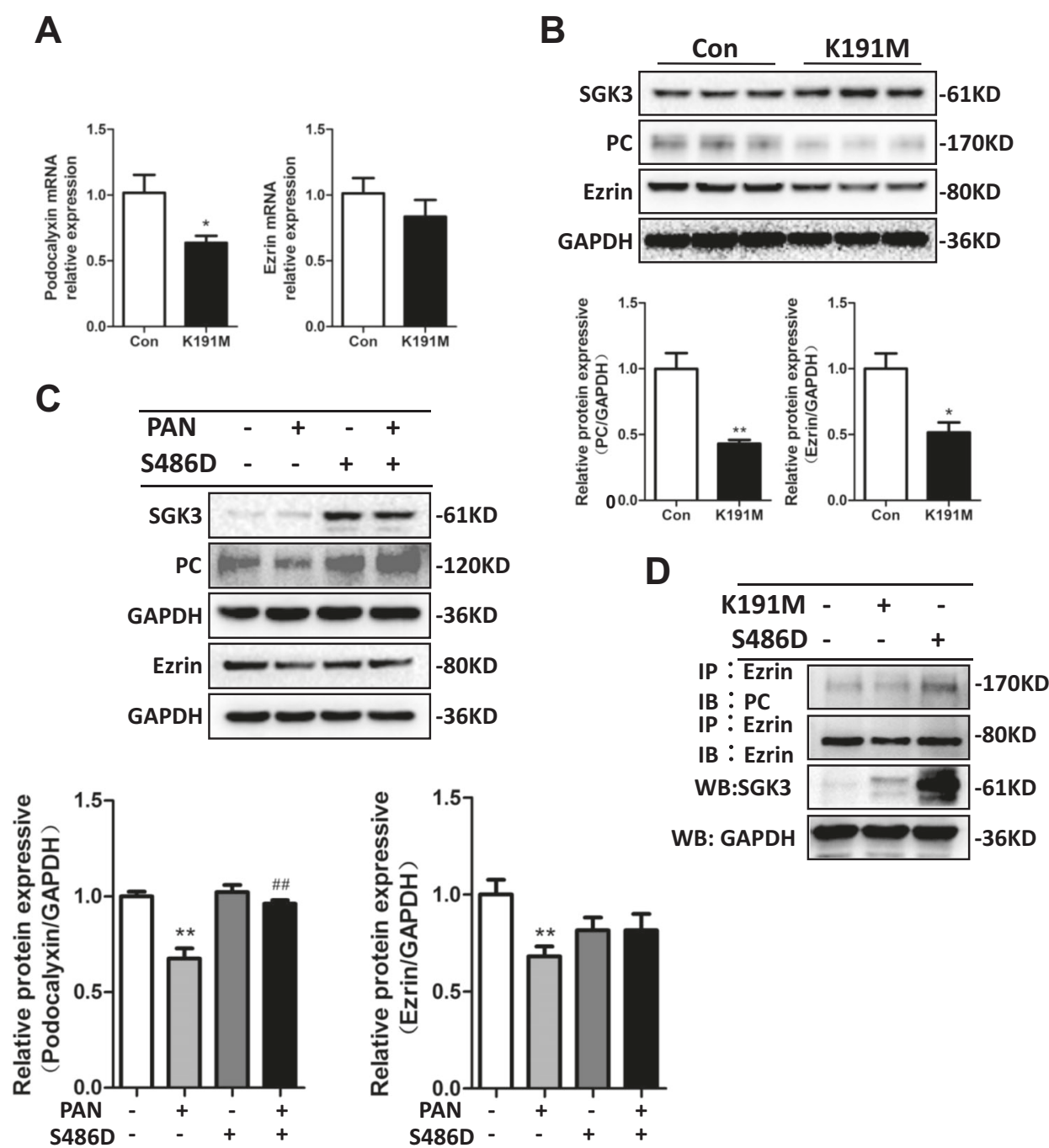

Fig. 4 SGK3-triggered PC and ezrin protein expression was involved in PAN-induced podocyte damage. a The scramble (control) or SGK3K191M plasmid were transiently transfected into cultured mouse podocytes for $48 \mathrm{~h}$. qRT-PCR was used to detect the mRNA expression levels of PC and ezrin. $\mathbf{b}$ Upper panel: immunoblot of PC and ezrin expression; GAPDH was used for normalization. Bottom panel: densitometric quantification of $P C$ and ezrin. Data are means \pm SEs of three independent experiments. ${ }^{*} P<0.05$ vs. the control group, ${ }^{* *} P<0.01$ vs. the control group. $\mathbf{c}$ The scramble (control) or SGK3-S486D plasmid were transiently transfected into cultured mouse podocytes for $48 \mathrm{~h}$. The podocytes were exposed to $50 \mathrm{\mu g} / \mathrm{ml} \mathrm{PAN}$ for the last $24 \mathrm{~h}$ of transfection. Top panel: immunoblot showing expression of SGK3, PC, and ezrin; GAPDH was used for normalization. Bottom panel:

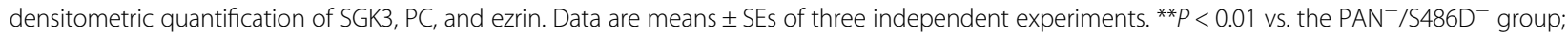
${ }^{\#} P<0.01$ vs. the $\mathrm{PAN}^{+} / \mathrm{S} 486 \mathrm{D}^{-}$group. $\mathbf{d}$ Mouse podocytes were transfected with the scramble (control) plasmid, SGK3-K191M, or SGK3-S486D plasmid in vitro. WCLs were precipitated with anti-ezrin antibody, and the immunoprecipitates were immunoblotted with anti-ezrin and anti-PC antibodies. SGK3 expression was measured in WCL immunoblots after SGK3-K191M or SGK3-S486D transfection

MG132 (Fig. 5a), indicating that SGK3 may regulate PC and ezrin expression through the proteasome pathway. Consistent with this, PC and ezrin ubiquitination levels were significantly higher in the SGK3-K191M-transfected mouse podocytes as compared to control mouse podocytes (Fig. 5b, c). These results suggested that, in the absence of SGK3 activity, PC and ezrin degradation is stimulated through the ubiquitin-proteasome degradation pathway.

\section{The SGK3/Nedd4-2 signaling pathway played a role in podocyte injury in vivo and in vitro}

To investigate why the lack of SGK3 activity increased PC and ezrin ubiquitination, we tested whether the SGK3 target protein Nedd4-2, a common E3 ubiquitin ligase, was involved in podocyte damage in vivo or in vitro. We first confirmed that SGK3 regulated Nedd4-2 phosphorylation (Fig. 6a). That is, the p-Nedd4-2/Nedd4-2 ratio decreased significantly in the SGK3-K191M- 

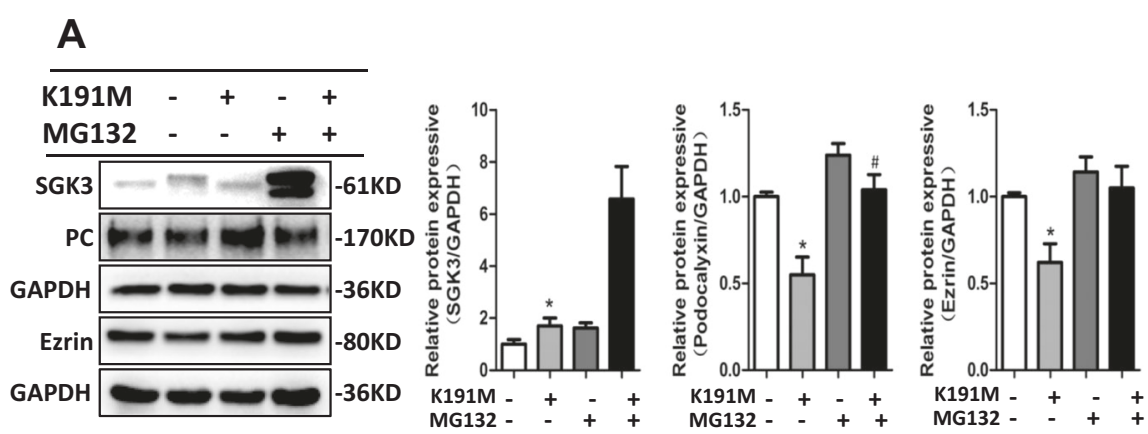

B
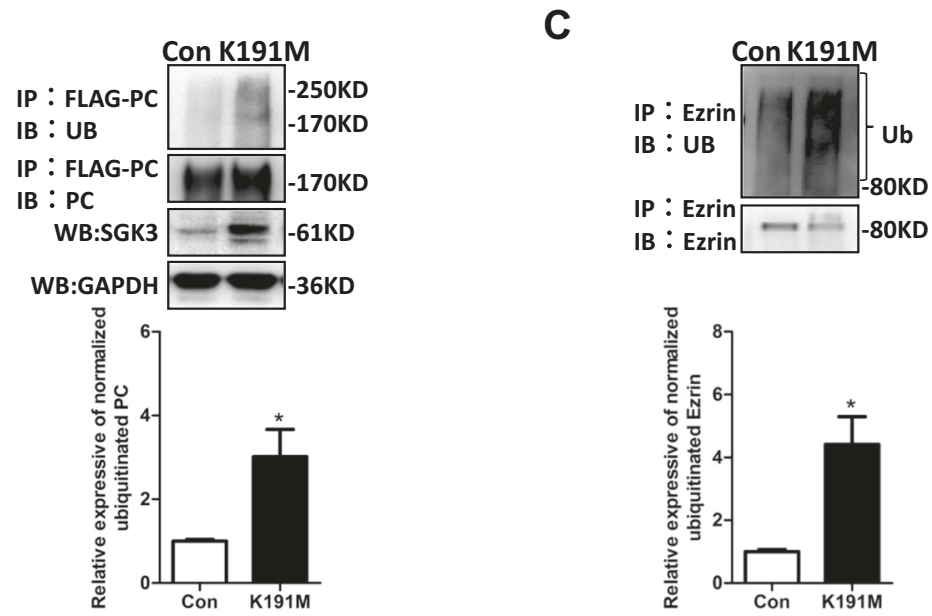

Fig. 5 SGK3 inactivation promotes the ubiquitin-mediated degradation of PC and ezrin in cultured mouse podocytes. Mouse podocytes were transfected with the scramble (control) or SGK3-K191M plasmid. After $40 \mathrm{~h}$ of transfection, cells were either untreated or treated with MG132 for additional $8 \mathrm{~h}$. a Left panel: immunoblot showing SGK3, PC, and ezrin expression; GAPDH was used for normalization. Right panel: densitometric quantification of SGK3, PC, and ezrin. Data are means \pm SEs of three independent experiments. ${ }^{*} P<0.05 \mathrm{vs}$. the K191 ${ }^{-} / \mathrm{MG} 132^{-}$-transfected group.

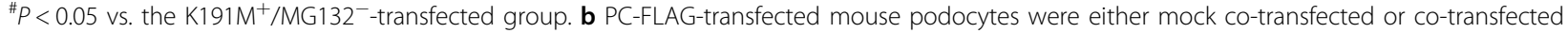
with the SGK3-K191M plasmid and then treated with MG132 for the last $8 \mathrm{~h}$ of transfection. Upper panel: WCLs were precipitated with the anti-FLAG antibody, and the immunoprecipitates were blotted with anti-PC and anti-Ub antibodies. WCL immunoblots were used to measure SGK3 expression after SGK3-K191M transfection. Bottom panel: The level of ubiquitinated ezrin was normalized to the immunoprecipitated PC. c Mouse podocytes were transfected with scramble (control) or SGK3-K191M plasmid for $48 \mathrm{~h}$ and then treated with MG132 for the last $8 \mathrm{~h}$ of transfection. Upper panel: WCLs were precipitated with anti-ezrin antibody, and western blots with anti-ezrin and anti-Ub antibodies were performed after the immunoprecipitates. Bottom panel: The level of ubiquitinated ezrin was normalized to the immunoprecipitated ezrin. Data are means \pm SEs of three independent experiments. ${ }^{*} P<0.05$ vs. control group

transfected mouse podocytes compared to the scramble plasmid-transfected mouse podocytes. We next verified the involvement of Nedd4-2 in podocyte damage both in vivo and in vitro. The $\mathrm{p}$-Nedd4-2/Nedd4-2 ratio decreased obviously in both the ADR nephritic mouse model and the PAN-induced podocyte injury cell model, implying a significant increase in Nedd4-2 activity and confirming the involvement of Nedd4-2 in podocyte damage (Fig. 6b, c). Finally, we investigated the role of the SGK3/Nedd4-2 signaling pathway in PAN-induced podocyte damage in vitro. The activity of Nedd4-2 was lower in $\mathrm{PAN}^{-} / \mathrm{S}_{486 \mathrm{D}^{+}}$-transfected mouse podocytes than in $\mathrm{PAN}^{-} / \mathrm{S}_{486 \mathrm{D}^{-}}$-transfected mouse podocytes (Fig. 6c). However, SGK3 activation completely reversed the PAN-induced decrease in the p-Nedd4-2/Nedd4-2 ratio (Fig. 6c). Therefore, our results strongly suggested that the SGK3/Nedd4-2 signaling pathway was involved in podocyte damage both in vivo and in vitro.

Nedd4-2 modulated ezrin protein expression, but not PC protein expression, through the ubiquitin-proteasome pathway in cultured mouse podocytes

To investigate whether the SGK3 target protein Nedd42 was involved in $\mathrm{PC}$ and ezrin expression and ubiquitination, we examined the effect of Nedd4-2 activation on $\mathrm{PC}$ and ezrin protein expression. Mouse podocytes were transfected with the wild-type (WT) Nedd4-2 plasmid or scramble plasmid for $48 \mathrm{~h}$. Transfected mouse podocytes 
A

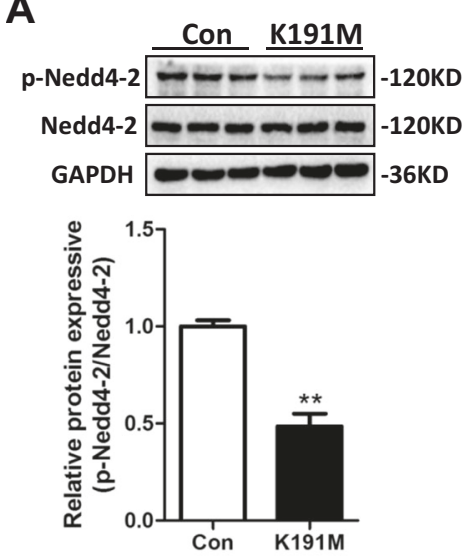

C

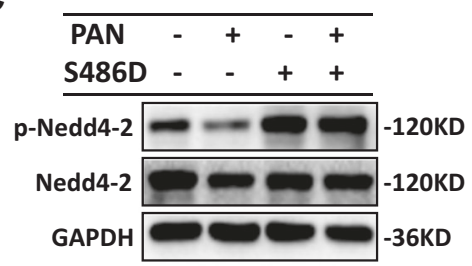

B
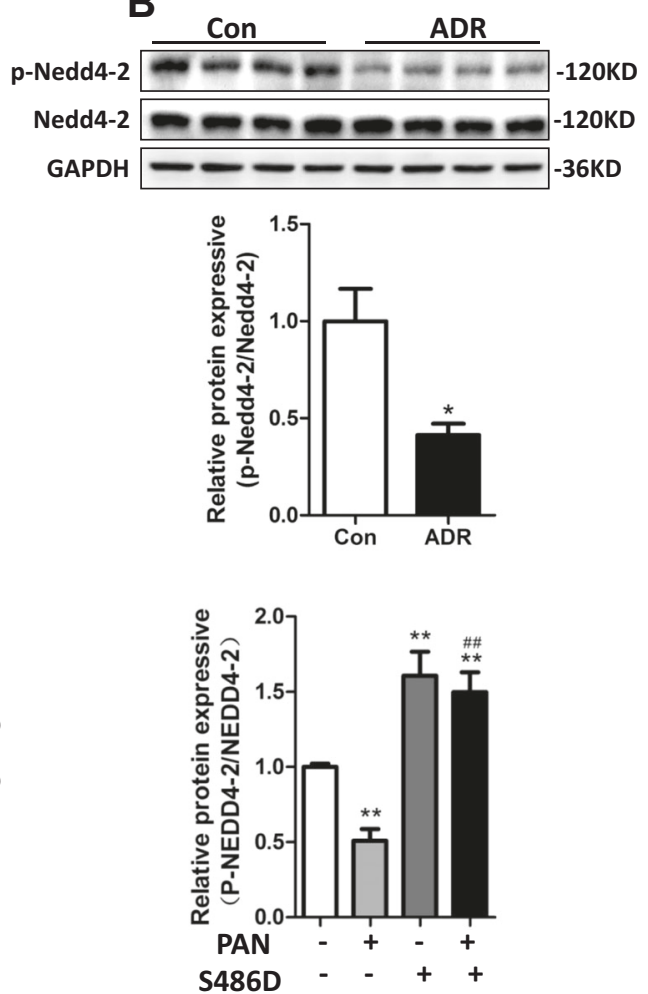

Fig. 6 The SGK3/Nedd4-2 signaling pathway was involved in podocyte damage both in vivo and in vitro. a Mouse podocytes were transfected with either the scramble (control) or the SGK3-K191M plasmid for $48 \mathrm{~h}$. Upper panel: immunoblot showing p-Nedd4-2 and Nedd4-2 expression; GAPDH was used for normalization. Bottom panel: densitometric quantification of the p-Nedd4-2/Nedd4-2 ratio. Data are means \pm SEs of three independent experiments. ${ }^{* *} P<0.01$ vs. the control group. $\mathbf{b}$ Upper panel: immunoblot showing p-Nedd4-2 and Nedd4-2 expression in control and ADR-injected mice; GAPDH was used for normalization. Bottom panel: Densitometric quantification of the p-Nedd4-2/Nedd4-2 ratio (6 samples/ group). ${ }^{*} P<0.01$ vs. the control mice. c The scramble or SGK3-S486D plasmid were transiently transfected into cultured mouse podocytes. Podocytes were treated with PAN for the last $24 \mathrm{~h}$ of transfection. Left panel: immunoblot showing p-Nedd4-2 and Nedd4-2 expression; GAPDH was used for normalization. Right panel: densitometric quantification of the $\mathrm{p}-\mathrm{Nedd} 4-2 / \mathrm{Nedd} 4-2$ ratio. Data are means $\pm \mathrm{SEs}$ of three independent experiments. ${ }^{* *} P<0.01$ vs. $\mathrm{PAN}^{-} / S 486 \mathrm{D}^{-}$-transfected group; ${ }^{\# \#} P<0.01$ vs. the $\mathrm{PAN}^{+} / S 486 \mathrm{D}^{-}$-transfected group

were then untreated or treated with MG132 for the last 8 $\mathrm{h}$ of transfection. Ezrin protein expression was significantly lower in the Nedd4- $2^{+} / \mathrm{MG} 132^{-}$-transfected mouse podocytes than in the Nedd4- $2^{-} / \mathrm{MG}_{132}{ }^{-}$-transfected mouse podocytes, while PC protein expression remained unchanged (Fig. 7a). Moreover, after administration of MG132, the decreased expression of ezrin in the Nedd4- $2^{+} / \mathrm{MG}_{132}{ }^{-}$group was partially reversed in the Nedd4- $2^{+} / \mathrm{MG} 132^{+}$group (Fig. 7a). Ezrin ubiquitination was significantly higher in WT Nedd4-2-transfected mouse podocytes than in the scramble plasmidtransfected mouse podocytes (Fig. 7b). These results indicated that Nedd4-2 downregulated ezrin, but not PC, through the ubiquitin-proteasome degradation pathway. All three Nedd4-2 short hairpin RNAs (shRNAs; \#1, \#2, and \#3), as well as the Nedd4-2 control shRNA, were used to infect mouse podocytes. Although two Nedd4-2 shRNAs (\#2 and \#3) visibly inhibited Nedd4-2, the
shRNA (\#2) had the highest Nedd4-2 inhibition efficiency and was thus selected for subsequent infections (data not shown). Silencing Nedd4-2 increased ezrin protein expression and increased ezrin ubiquitination (Fig. 7c, d). Together, our results strongly suggested that Nedd4-2 downregulated ezrin protein expression by increasing ezrin ubiquitination.

\section{SGK3-mediated Nedd4-2 activity affected the interaction between Nedd4-2 and ezrin}

As our results indicated that Nedd4-2 induced ezrin ubiquitination, we next tested whether SGK3-mediated Nedd4-2 activity affected the interaction between Nedd42 and ezrin. To test this, we performed Co-IP studies on HEK293T cells. HEK293T cells were transfected with WT Nedd4-2, with or without one of the plasmids SGK3K191M and SGK3-S486D. Transfection with SGK3K191M led to increased Nedd4-2 activity and decreased 
A
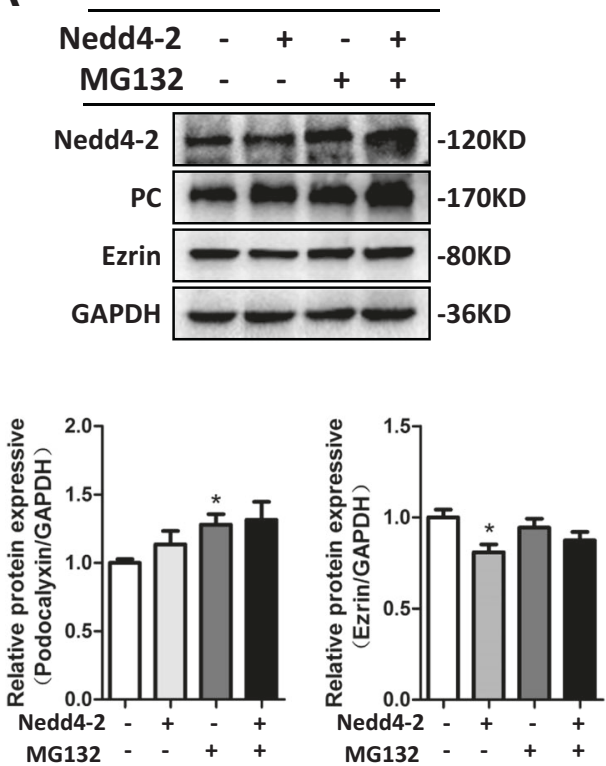

C
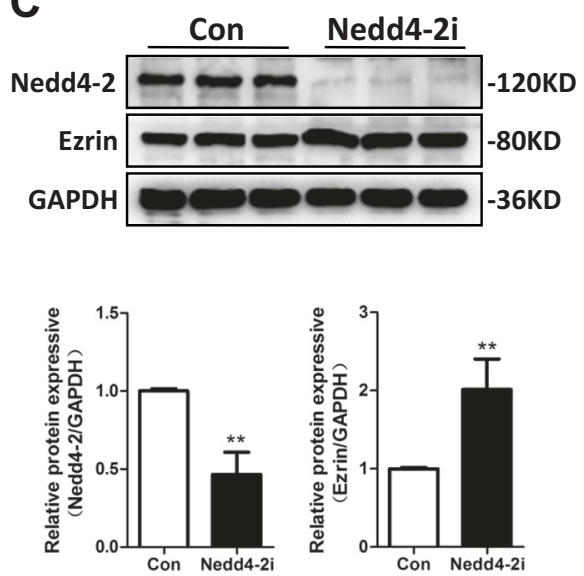

B
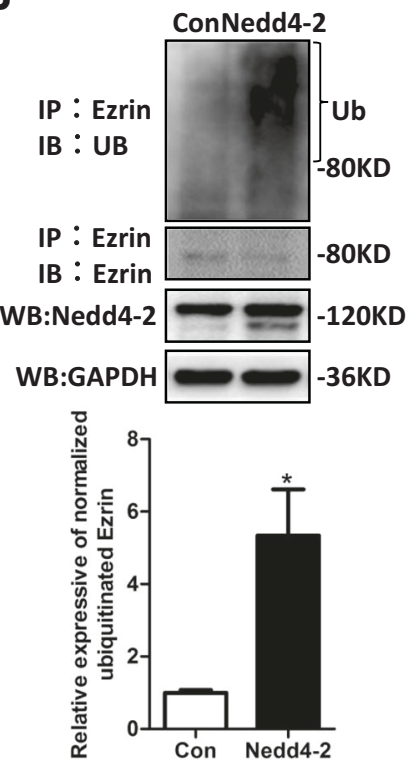

D

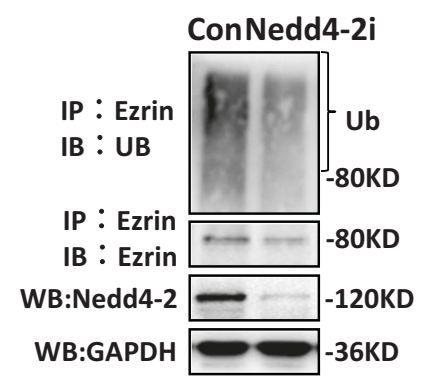

Fig. 7 Nedd4-2 modulated ezrin ubiquitination but not PC ubiquitination. Mouse podocytes were transfected with either the pcDNA3 (Con) or the WT Nedd4-2 plasmid for $48 \mathrm{~h}$. Cells were either untreated or treated with MG132 for the last $8 \mathrm{~h}$ of transfection. a Upper panel: immunoblot showing Nedd4-2, PC, and ezrin expression; GAPDH was used for normalization. Bottom panel: densitometric quantification of PC and ezrin. ${ }^{*} \mathrm{P}<0.05$ vs. Nedd4-2/MG132-group. b WCLs were precipitated with the anti-ezrin antibody, and the immunoprecipitates were immunoblotted with anti-ezrin and anti-Ub antibodies. Upper panel: immunoblots were used to measure Nedd4-2 expression in WCLs after transfection with the WT Nedd4-2 plasmid. Bottom panel: The level of ubiquitinated ezrin was normalized to the immunoprecipitated ezrin. Data are means \pm SEs of three independent experiments. ${ }^{*} P<0.05$ vs. control group. c Mouse podocytes were infected with scramble (control) or Nedd4-2-specific shRNAs for $72 \mathrm{~h}$. Top panel: immunoblot showing Nedd4-2 and ezrin expression; GAPDH was used for normalization. Bottom panel: densitometric quantification of Nedd4-2 and ezrin. Data are means \pm SEs of three independent experiments; for each sample, three wells were treated. ${ }^{*} P<0.01$ vs. the control group. $\mathbf{d}$ Mouse podocytes were transfected with recombinant lentiviral Nedd4-2-eGFP shRNA or scramble (control) lentiviral shRNA. WCLs were precipitated with the anti-ezrin antibody, and the immunoprecipitates were immunoblotted with anti-ezrin and anti-Ub antibodies. Nedd4-2 expression in WCLs was measured with immunoblot assays after Nedd4-2 shRNA transfection

ezrin expression, while transfection with SGK3-S486D led to decreased Nedd4-2 activity (Fig. 8a). As ezrin harbors PY, the motif targeted by Nedd4-2, it was unsurprising that Nedd4-2 and ezrin interacted in HEK293T cells under basic conditions (Fig. 8b). After transfection with SGK3-K191M and WT Nedd4-2, Nedd4-2 and ezrin interaction increased in HEK293T cells, while Nedd4-2 and ezrin interaction decreased after transfection with SGK3-S486D and WT Nedd4-2 (Fig. 8b). Therefore, increased Nedd4- 2 activity mediated by the inactivation of SGK3 also increased the interaction between Nedd4-2 and ezrin. 
A

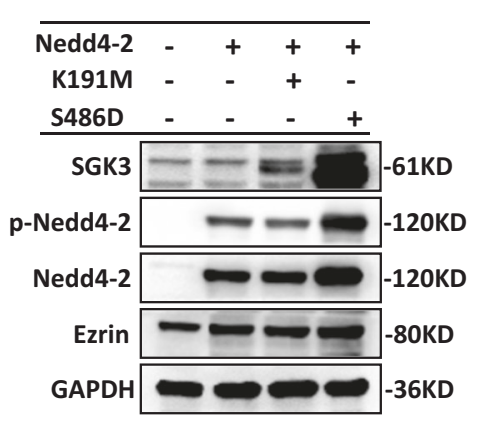

B

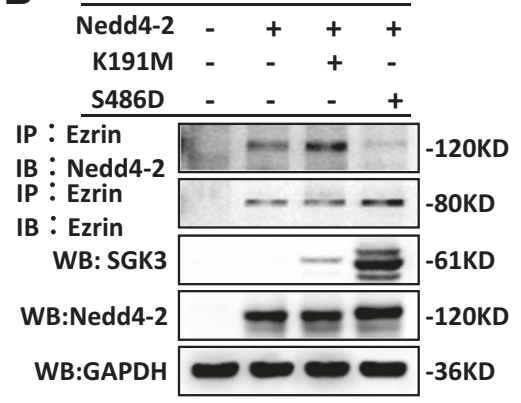

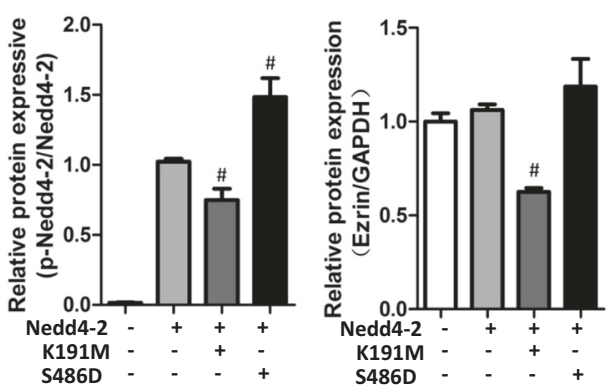

Fig. 8 SGK3-mediated Nedd4-2 phosphorylation affected the interaction between Nedd4-2 and ezrin. a Upper panel: HEK293T cells were transfected with the indicated plasmids. Immunoblot analysis of SGK3, P-Nedd4-2, Nedd4-2, and ezrin expression; GAPDH was used for normalization. Bottom panel: densitometric quantification of the P-Nedd4-2/Nedd4-2 ratio and ezrin. Data are means \pm SEs of three independent experiments. ${ }^{\#} P<$ 0.05 vs. cells transfected with Nedd4-2 $2^{+} / \mathrm{K} 191 \mathrm{M}^{-} / \mathrm{S}_{486 \mathrm{D}^{-}}$. $\mathbf{b}$ WCLs were precipitated with the anti-ezrin antibody, and the immunoprecipitates were blotted with anti-ezrin and anti-Nedd4-2 antibodies. SGK3 and Nedd4-2 expression levels in the WCLs were measured with immunoblot assays

SGK3 and the SGK3/Nedd4-2 signaling pathway are involved in the regulation of the ezrin/PC interaction

To explore whether SGK3 and the SGK3/Nedd4-2 signaling pathway regulated the stability of the PC/ezrin complex, we performed Co-IP assays in HEK293T cells. To do this, we first transfected the SGK3-K191M plasmid into HEK293T cells expressing PC-FLAG. We detected three bands in the PC-FLAG-transfected HEK293 cells. PC, but not ezrin, protein expression was downregulated in the transfected HEK293T cells, as compared to control cells (Fig. 9a). As Nedd4-2 is not expressed in HEK293 cells $^{32,33}$, these results suggested that SGK3-mediated ezrin expression depended on Nedd4-2 but that SGK3mediated PC expression did not. Moreover, the inactivation of SGK3 decreased PC expression, directly reducing the interaction between $\mathrm{PC}$ and ezrin (Fig. 9a).

We next transfected the HEK293T cells expressing PCFLAG with the WT Nedd4-2 plasmid. Ezrin protein expression was downregulated after WT Nedd4-2 plasmid transfection; PC protein expression remained constant (Fig. 9b). The interaction between PC and ezrin in the cells transfected with WT Nedd4-2 was significantly lower than that in the control cells. This indicated that the activation of Nedd4-2 decreased ezrin expression, but not PC expression, contributing to the decreased interaction between PC and ezrin. In addition, we found that ezrin co-precipitated with PC in the PC-FLAG-transfected
HEK293T cells, indicating that exogenously expressed PC interacts with endogenous ezrin in HEK293T cells, and suggesting that this interaction does not require the co-expression of additional podocyte-specific proteins (Fig. 9a, b).

Finally, we examined the role of the SGK3/Nedd42 signaling pathway with respect to the PC/ezrin interaction. To do this, HEK293T cells expressing PC-FLAG were co-transfected with WT Nedd4-2 and either the SGK3-K191M or the SGK3-S486 plasmid. The PC/ezrin interaction was reduced in cells transfected with SGK3K191M as compared to control cells, while the PC/ezrin interaction increased in cells transfected with SGK3S486D as compared to control cells (Fig. 9c). As SGK3 targets Nedd4-2, our results suggested that SGK3 and the SGK3/Nedd4-2 signaling pathway were both involved in regulating the stability of the PC/ezrin complex.

\section{Discussion}

SGK3, an important downstream target protein of phosphatidylinositol-3-kinase, is involved in the regulation of substrate transport ${ }^{7}$, as well as cell growth, survival, and proliferation ${ }^{34,35}$. Previously, we showed that SGK3 deficiency leads to decreased podocin expression and reduced podocyte viability ${ }^{4}$. However, the signaling mechanisms involved in SGK3-triggered podocyte function are not well known. Here we found that PC and ezrin 


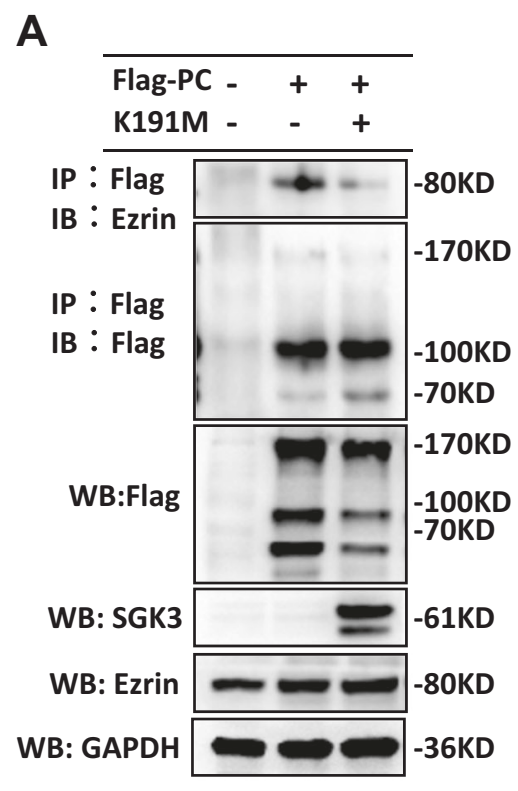

C

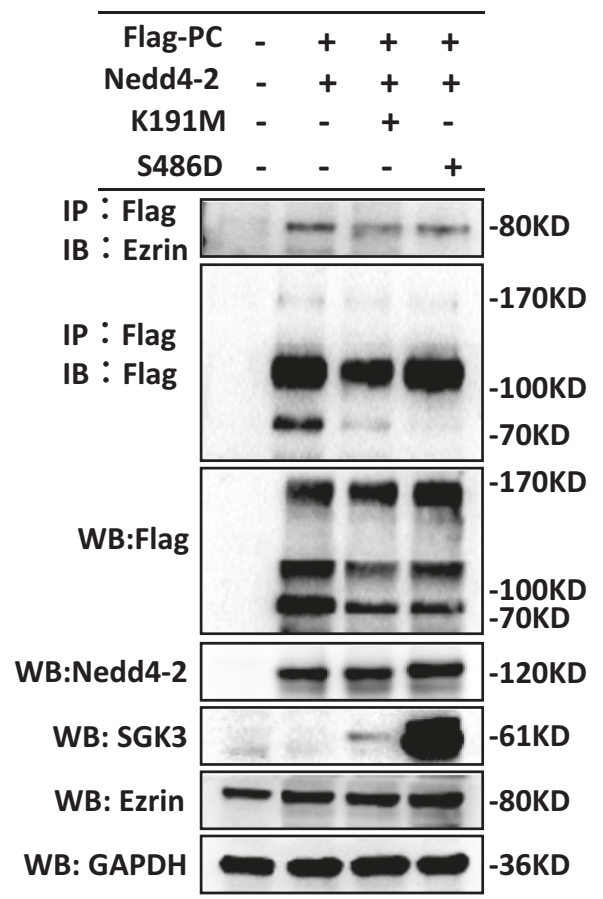

B

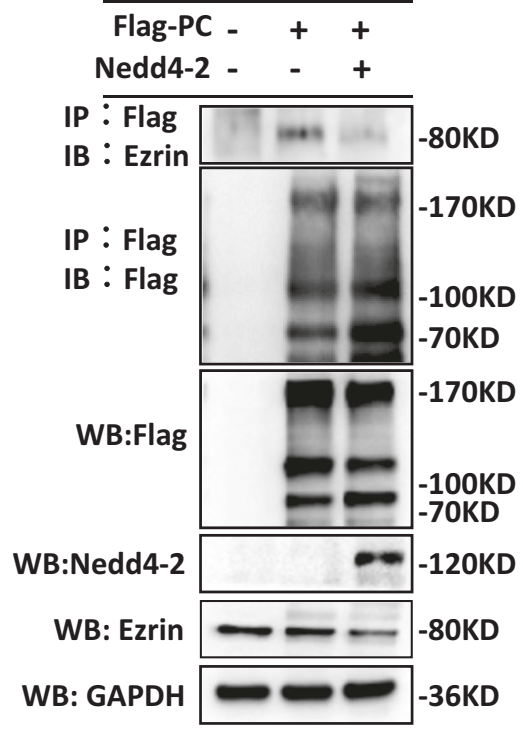

Fig. 9 SGK3 and the SGK3/Nedd4-2 signaling pathway were involved in the regulation of PC/ezrin interaction. a HEK293T cells were mock transfected or transfected with the SGK3-K191M plasmid for $24 \mathrm{~h}$. WCLs were precipitated with anti-FLAG antibodies and then blotted with anti-FLAG and anti-ezrin antibodies. FLAG, SGK3, and ezrin expression levels were measured in the WCLs with immunoblot assays. b pc-FLAG-transfected HEK293T cells were mock co-transfected or co-transfected with the WT Nedd4-2 plasmid for $24 \mathrm{~h}$. Immunoblots of the WCLs were precipitated with anti-FLAG antibodies and blotted with anti-FLAG and anti-ezrin antibodies. FLAG, Nedd4-2, and ezrin expression levels in the WCLs were measured with immunoblot assays. c HEK293T cells were transfected with the indicated plasmids for $24 \mathrm{~h}$. Immunoblots of the WCLs were precipitated with the anti-FLAG antibody, and blotted with anti-FLAG and anti-ezrin antibodies. FLAG, SGK3, Nedd4-2, and ezrin expression levels in the WCLs were measured with immunoblot assays 
protein expression levels were reduced after podocyte damage, both in vivo and in vitro. We demonstrated that both PAN treatment and SGK3 inactivation resulted in increased PC and ezrin ubiquitination and that SGK3 activation partially reversed PAN-induced decreases in PC and ezrin protein expression. Moreover, the enhancement of Nedd4-2 activity via SGK3 inactivation increased ezrin ubiquitination but not PC ubiquitination. Our results suggested that SGK3 and the SGK3/Nedd4-2 signaling pathway helped to maintain the stability of the PC/ezrin complex in vitro.

One intriguing finding of our study was that decreased PC and ezrin protein expression was not caused by reduced PC and ezrin mRNA transcription after PAN-induced podocyte damage. Rather, this decreased protein expression primarily resulted from the increased PC and ezrin protein degradation induced via the ubiquitinproteasome pathway. Ezrin, a plasma membrane-cytoskeleton linker protein, mainly regulates cell adhesion and morphogenesis ${ }^{36}$. Previous studies have shown that ezrin activity and/or expression decreases in animal models of glomerular disease ${ }^{28,37}$ and in nephropathic patients ${ }^{29,37}$. Consistent with these studies, we found that ezrin was downregulated after podocyte damage both in vivo and in vitro, suggesting that decreased ezrin expression may be a marker for podocyte damage. PC is the most highly sialylated glycoprotein in podocytes ${ }^{13,23}$. Several studies have reported the downregulation of PC in ADR models ${ }^{38-40}$ and upregulation of PC mRNA expression in mouse podocytes after PAN-induced podocyte damage ${ }^{41,42}$. Both of these findings were consistent with our results. Incongruent with our results, some studies have shown that PC protein expression increased in mouse podocytes with PAN-induced podocyte damage ${ }^{41,42}$, but this discrepancy was probably due to differences in experimental methods (e.g., PAN treatment period or concentration).

We previously implicated SGK3 in the mediation of PAN-induced podocyte damage ${ }^{4}$, but the mechanisms underlying this mediation remained unclear. Here we linked SGK3 activation to PC and ezrin protein expression after podocyte damage, both in vivo and in vitro. The downregulation of SGK3 not only suppressed PC mRNA transcription but also inhibited $\mathrm{PC}$ and ezrin protein expression. In addition, SGK3 activation mostly reversed the PAN-induced inhibition of $\mathrm{PC}$ and ezrin protein expression. Our results therefore convincingly indicated that SGK3 affected PAN-induced podocyte damage by triggering $\mathrm{PC}$ and ezrin protein expression. It should be noted that SGK3 inactivation decreased PC mRNA expression, while PAN treatment increased PC mRNA expression, suggesting that PAN-induced podocyte damage did not depend solely on SGK3 activity.
The mechanisms underlying this contradiction (i.e., that PAN treatment led both to SGK3 inactivation and to increased PC mRNA expression) are still unclear and merit further investigation.

We have shown here, for the first time, that SGK3 activation triggers the ubiquitin-mediated degradation of PC and ezrin. This novel result provides insight into the pathomechanism of SGK3 in podocytes. It is well known that PC and ezrin are regulated by a variety of factors, including phosphorylation, binding partners ${ }^{28}$, and ubiquitination ${ }^{43}$. A recent study showed that ezrin was ubiquitinated by WWP1, a HECT ubiquitin ligase ${ }^{26}$ that mediates the alteration of ezrin function, but does not degrade ezrin ${ }^{44}$. However, the ubiquitination of PC has not previously been reported. Herein we found that SGK3 inactivation led to PC and ezrin protein degradation, primarily via the proteasome pathway. Interestingly, we found that the SGK3-targeted protein Nedd4-2 mediated ezrin ubiquitination, but not $\mathrm{PC}$ ubiquitination, indicating that SGK3 modulates $\mathrm{PC}$ and ezrin degradation through different regulatory mechanisms. This result was consistent with the known protein structures, in that there is a PY motif in ezrin but not in PC. The PY motif can be bound, and therefore regulated, by Nedd4-2. Consistent with this, SGK3 phosphorylates Nedd4-2 and regulates the binding of Nedd4-2 with its target protein, ezrin (Fig. 8). It should be stressed that the molecular details of SGK3-mediated PC ubiquitination remain obscure. In addition, unlike WWP1mediated ezrin ubiquitination, Nedd4-2-mediated ezrin ubiquitination decreased ezrin protein expression.

Several studies have shown that structural and/or functional defects in either PC or the associated cytoskeletal linker proteins may result in glomerular disorders ${ }^{17}$. Disruption of the $\mathrm{PC} /$ ezrin interaction in glomerular epithelial cells is associated with the dephosphorylation of ezrin, the neutralization of the negative PC surface charge ${ }^{17,24}$, and the decreased protein expression of either PC or ezrin ${ }^{24}$. Here we found that the deceased SGK3 activity resulted in decreased PC and ezrin protein expression, consistent with the disrupted binding of PC and ezrin in SGK3-inactivated mouse podocytes and HEK293T cells. SGK3 downregulation led to decreased PC mRNA expression and to increased PC and ezrin protein degradation. Together, these effects might disrupt the PC/ezrin interaction. Notably, Nedd4-2 activity did not influence PC expression. This indicated that the decreased stability of the PC/ezrin complex induced by the decrease in SGK3 activity was not completely dependent on the SGK3/Nedd4-2 signaling pathway. Several studies have focused on the role of AKT in the kidney ${ }^{8-10}$. AKT is highly structurally and functionally homologous with SGK3 ${ }^{11}$. AKT2 maintains podocyte viability and function via the $\mathrm{AKT} / \mathrm{mammalian}$ 


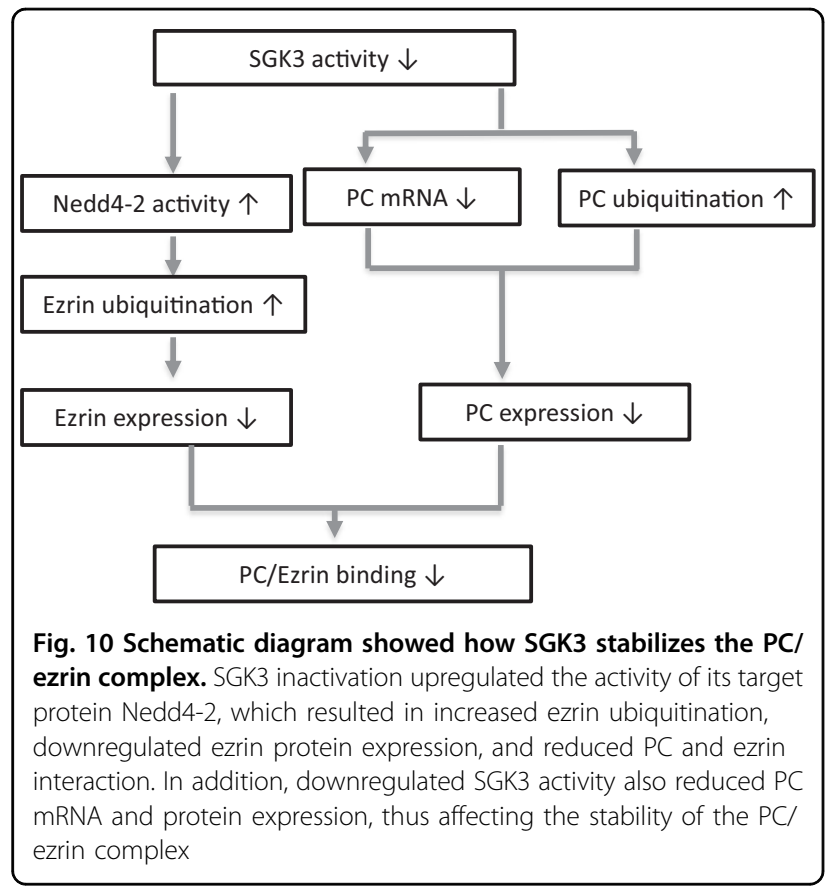

target of rapamycin or the AKT/GSK3 $\beta$ pathways ${ }^{10,45}$. SGK3 might regulate PC expression through the similar AKT pathway, and indeed, other pathways underlying podocyte dysfunction might be induced by a decrease in SGK3 activity.

In summary, SGK3 inactivation played an important role in the regulation of $\mathrm{PC}$ and ezrin expression. Here we demonstrated that SGK3 inactivation downregulated PC mRNA and upregulated the ubiquitin-mediated degradation of PC and ezrin, thereby inhibiting the protein expression of PC and ezrin. These changes destabilized the PC/ezrin complex. Moreover, SGK3-triggered Nedd42 activity degraded ezrin rather than PC (Fig. 10). Collectively, our results suggested that the regulation of SGK3 and the SGK3/Nedd4-2 signaling pathway determines the stability of the podocyte PC/ezrin complex.

\section{Methods and materials \\ Reagents and antibodies}

Polyclonal rabbit anti-SGK3, anti-Nedd4-2 and antiphospho-Nedd4-2 (Ser342) antibodies were purchased from Cell Signaling Technology, Inc. (Beverly, MA, USA). Polyclonal rabbit anti-ezrin was purchased from Abcam (Cambridge, MA, USA). Polyclonal goat anti-PC antibody was purchased from Research \& Diagnostics Systems, Inc. (Minneapolis, MN, USA). Polyclonal rabbit anti-Desmin (I-448) was purchased from Bioworld Technology, Co. Ltd. (Nanjing, China). Monoclonal mouse anti-GAPDH antibody; polyclonal rabbit anti-flag tag antibody; and horseradish peroxide-conjugated anti-rabbit, anti-mouse, and anti-goat secondary antibodies were purchased from
Proteintech (Wuhan, China). Monoclonal mouse antiubiquitin antibody was purchased from Covance Inc. (UT, USA). The pcDNA3/mNedd4-2, pMO/mSGK3-K191M (SGK3-K191M), and $\mathrm{pMO} / \mathrm{mSGK3-S486D} \mathrm{(SGK3-}$ S486D) plasmids were constructed as previously described $^{4}$. The PC-Myc-DDK plasmid (Flag-epitope at $\mathrm{N}^{-}$terminal of mouse $\mathrm{PC}$ ) was purchased from Origene Technologies, Inc. (Rockville, MD, USA). The plasmid of the mouse Flag-tagged WT Ub was provided by Dr. Qiuhong Duan (Department of Biochemistry and Molecular Biology, Tongji Medical College, Huazhong University of Science \& Technology, Wuhan, China). Three recombinant lentiviruses against Nedd4-2 were designed and generated by Genechem (Shanghai, China). PAN was purchased from Sigma Chemical (St Louis, MO, USA). MG132 was purchased from Selleck (Shanghai, China). A/ $G$ plus agarose for immunoprecipitation (IP) assay was purchased from Beyotime Biotechnology (Shanghai, China). Phosphatase inhibitor mixture and proteinase inhibitor cocktail were purchased from Roche Applied Science (Indianapolis, IN).

\section{ADR nephritis mouse model}

All experiments were performed in accordance with the National Institutes of Health guidelines for the use and care of experimental animals and were approved by the Animal Care and Use Committee of Tongji Medical College. The generation and characters of ADRinduced nephrosis model were previously described ${ }^{4}$. Briefly, male mice (8-week-old), body weight $20-25 \mathrm{~g}$, were treated with a single dose of $10.5 \mathrm{mg} / \mathrm{kg}$ ADR (Sigma, USA, D1515) by tail vein injection (ADR group, $n=6)$. The mice in the control group were administered with an equivalent volume of phosphate-buffered saline (PBS) (control group, $n=6$ ). On day 14, the mice were sacrificed under anesthesia and both blood samples and kidneys were collected. The harvested kidneys were collected for western blotting and immunohistochemical analysis.

\section{Histochemistry and immunohistochemistry}

PAS staining and immunohistochemistry studies proceeded and analyzed essentially as previously described ${ }^{4}$. In short, paraffin-embedded mouse kidney sections (5- $\mu \mathrm{m}$ thickness) for PAS and immunohistochemical staining were performed using routine protocol. Sections were stained with PAS reagent by standard procedure. Immunohistochemistry staining was performed using routine protocol. The slides were incubated with rabbit polyclonal anti-ezrin antibody and goat anti-PC antibody overnight at $4{ }^{\circ} \mathrm{C}$, followed by incubation with polyperoxidase anti-rabbit and anti-goat IgG secondary antibodies for $30 \mathrm{~min}$ at room temperature. Staining was then performed using a DAB Kit. Histological 
examination was performed via light microscopy. Positive expression for PC and ezrin were semi-quantitative using the Image Pro Plus software.

\section{Urine and serum biochemistry}

Blood urea nitrogen and urinary and serum creatinine levels were determined by using the respective kits (NINGBO ELEJECH Biological Technology Co., LTD, Zhejiang, China), according to the manufacturer's protocols. Urinary albumin was standardized to urinary creatinine and expressed as $\mathrm{mg} / \mathrm{mg}$ Ucr.

\section{Cell culture, transfection, and recombinant lentivirus infection}

The conditional immortalized mouse podocytes were handled as described earlier ${ }^{4}$. Briefly, cells were maintained in RPMI 1640 medium (HyClone, USA) supplemented with $10 \%(\mathrm{v} / \mathrm{v})$ fetal bovine serum (GIBCO, Thermo Fisher Scientific Inc. USA), $100 \mathrm{U} / \mathrm{ml}$ penicillin, and $100 \mu \mathrm{g} / \mathrm{ml}$ streptomycin. To propagate podocytes, cells were cultivated on type I collagen at $33{ }^{\circ} \mathrm{C}$ under $5 \%$ $\mathrm{CO}_{2}$ and $95 \%$ air in culture medium supplemented with $10 \mathrm{U} / \mathrm{ml}$ recombinant interferon- $\gamma$ (IFN- $\gamma$ ). To induce differentiation, podocytes were transferred at $37^{\circ} \mathrm{C}$ in IFN- $\gamma$-free medium. Mouse podocytes between passages 9 and 20 were used in all experiments. HEK293T cell cultures were maintained at $37^{\circ} \mathrm{C}$ in Dulbecco's modified Eagle's medium (HyClone, USA), supplemented with $10 \%$ fetal bovine serum, $100 \mathrm{U} / \mathrm{ml}$ penicillin, and $100 \mu \mathrm{g} / \mathrm{ml}$ streptomycin. Mouse podocytes and HEK293T cells were transiently transfected with various plasmids and respective scramble plasmids using Lipofectamine 2000 reagent (Invitrogen, Carlsbad, CA) according to the manufacturer's protocol for adherent cells. Cells were analyzed 24-48 $\mathrm{h}$ post transfection. Mouse podocytes were infected with scramble or Nedd4-2-specific lentiviruses. Seventytwo hours after shRNA infection, western blotting was used to evaluate the suppression of Nedd4-2 expression in different cell groups.

\section{Cell viability assay}

Cell viability was assessed by an MTT assay. Briefly, mouse podocytes were seeded into 96 -well plates at a density of $2 \times 10^{3} /$ well followed by treatment with or without PAN administration for $24 \mathrm{~h}$. In all, $10 \mu \mathrm{l} \mathrm{of} 5 \mathrm{mg} /$ $\mathrm{ml}$ MTT solution in PBS was added without removal of culture medium followed by incubation at $37^{\circ} \mathrm{C}$ for $4 \mathrm{~h}$. The medium was removed and $100 \mu \mathrm{l}$ of dimethyl sulfoxide was added to dissolve the formazan crystals for about $15 \mathrm{~min}$ by a shaker. Absorbance of each well was measured by a microplate reader (BioTek, Winooski, VT, USA) at $570-\mathrm{nm}$ wavelength, followed by calculating the mean value. All data were determined from three independent cultures.

\section{Immunofluorescence staining and and confocal microscopy}

Mouse podocytes grown on glass coverslips were fixed with $4 \%$ paraformaldehyde for $30 \mathrm{~min}$ and permeabilized with $0.1 \%$ Triton X-100 in PBS for 5 min. After blocking with $10 \%$ donkey serum for $30 \mathrm{~min}$, the slides were immunostained with primary antibodies against PC and ezrin. The slides were then stained with IFKine greenconjugated donkey anti-goat antibody (\#A24231, Abbkine) and Alexa Fluor 594-conjugated donkey anti-rabbit antibody (127803, Jackson). Cells were costained with 4,6diamidino-2-phenylindole (blue). For F-actin staining, slides was stained with rhodamine-phalloidin (Invitrogen, Carlsbad, CA) for $20 \mathrm{~min}$ at room temperature. Images were acquired using a Leica TCS-SL confocal microscope.

\section{Western blot analysis}

Protein expression was analyzed by western blot analysis as described previously ${ }^{4}$. In brief, protein extracts from the kidneys as well as whole-cell protein from stable cell lines were used. Equal amounts of proteins were loaded on sodium dodecyl sulfate-polyacrylamide gel electrophoresis (SDS-PAGE) gels for separation and transferred onto polyvinylidene difluoride membrane (Millipore Corp., Bedford, MA). Membranes were then blocked for $1 \mathrm{~h}$ at room temperature using 5\% skim milk and $0.1 \%$ Tween 20 in Tris-buffered saline. The immunoblot was then performed overnight at $4{ }^{\circ} \mathrm{C}$ using appropriate primary antibodies. Protein signals were detected using the corresponding horseradish peroxidaseconjugated secondary antibody and enhanced chemiluminescence (Millipore, Germany). All experiments were performed in triplicate, and the blots of p-Nedd4-2 and Nedd4-2 were taken from the same membrane.

\section{Real-time RT-PCR analysis}

Total RNA isolation and quantitative RT-PCR (qRTPCR) were carried out by a routine procedure. Total RNA was isolated from incubated cells using TRIzol Reagent (Invitrogen, Thermo Fisher Scientific Inc. USA) according to the manufacturer's instructions. The first-strand cDNA was carried out using a ReverAid First Strand cDNA Synthesis Kit (Thermo, Thermo Fisher Scientific Inc. USA) according to the manufacturer's protocol. Real-time RT-PCR was performed on the CFX96 Touch $^{\text {TM }}$ Real-Time PCR Detection System (Bio-rad, Hercules, CA, USA). The PCR results for each sample were normalized using glyceraldehyde 3-phosphate dehydrogenase (GAPDH) mRNA as an internal control. The $\Delta \Delta \mathrm{Ct}$ technique was used to calculate cDNA content in each sample. Primers for PC and ezrin were designed based on the GenBank accession numbers (PC: NM_013723, ezrin: NM_22350). The sequences of the primer pairs used in RT-PCR are as follows: mouse PC forward: CTGTAAGCGACCT 
GAACCGT, backward: TGCTGCCACCTTGATGTCTA; mouse ezrin forward: TCACAGAGGCAGAGAAGA ATG, backward: TCTCGTTGTGGATGATGTCA; and mouse GAPDH forward: GTCAAGCTCATTTCCTGG TATG, backward: CCTCTCTTGCTCAGTGTCCTT. All experiments were performed in triplicate.

\section{Ubiquitination assay in mouse podocytes}

Ubiquitination assays were performed as described ${ }^{32}$. Mouse podocytes were treated with $50 \mu \mathrm{g} / \mathrm{ml}$ PAN or transfected with expression constructs (as shown in the figures). To inhibit proteasomal degradation, $10 \mu \mathrm{M}$ MG132 was added to mouse podocytes $8 \mathrm{~h}$ before lysis. Twenty-four hours after PAN treatment or $48 \mathrm{~h}$ after transfection, cells were washed with PBS and dissociated in $1 \mathrm{ml}$ dissociation buffer [ $5 \%$ glycerol, $1 \mathrm{mM}$ EDTA, and $1 \mathrm{mM}$ EGTA ( $\mathrm{pH}$ 8.0)]. Then cells were washed with $10 \mathrm{ml}$ PBS and centrifuged at $4{ }^{\circ} \mathrm{C}, 3000 \mathrm{rpm}$, for $15 \mathrm{~min}$. Cell pellets were frozen at $-70^{\circ} \mathrm{C}$ for $2-4 \mathrm{~h}$. Pellets were then resuspended in $1 \mathrm{ml}$ lysis buffer [50 mM HEPES (pH 7.5), $1 \mathrm{mM}$ EGTA, $150 \mathrm{mM} \mathrm{NaCl}, 1.5 \mathrm{mM} \mathrm{MgCl}$, $10 \%$ glycerol, $1 \%$ TritonX-100, $25 \mathrm{mM}$ sodium fluoride, $40 \mathrm{mM} \beta$-glycerolphosphate, $10 \mathrm{mM}$ sodium pyrophosphate] containing phenylmethanesulfonylfluoride, cocktail, and phosphatase inhibitors. The lysates were centrifuged at $13,000 \mathrm{rpm}\left(4^{\circ} \mathrm{C}\right)$ for $15 \mathrm{~min}$. The supernatant proteins (about $1.5 \mathrm{mg}$ ) were subjected to IP using the appropriate primary antibody (anti-ezrin antibody, anti-Flag antibody) and protein A/G agarose beads overnight at $4{ }^{\circ} \mathrm{C}$. Sample loading buffer $5 \times$ was added to the immunoprecipitates and boiled for $10 \mathrm{~min}$. The samples, centrifuged at 13,000 rpm for $5 \mathrm{~min}$, were separated by SDS-PAGE for western blot analysis and immunoblotted with anti-ubiquitin or anti-PC antibody. Scanning densitometry was measured as described above.

\section{Co-IP assays}

Mouse podocytes were maintained and administered with PAN for $24 \mathrm{~h}$ or transfected with appropriate expression constructs for $48 \mathrm{~h}$. HEK293T cells, grown on $10-\mathrm{cm}$ dishes, were transfected with $10 \mu \mathrm{g}$ of the plasmids DNA encoding FLAG-tagged PC, mouse Nedd4-2, mouse SGK3-S486D, mouse SGK3-K191M, and/or empty vector using Lipofectamine 2000 reagent for $24 \mathrm{~h}$. After treatment and transfection, cells were dissociated in $500 \mu \mathrm{l}$ IP lysis buffer (Beyotime Biotechnology, Shanghai, China). Proteins (about $1.0 \mathrm{mg}$ ) were incubated with the appropriate primary antibody (anti-ezrin antibody, anti-Flag antibody) and protein A/G agarose beads. Then the samples were subjected to western blot analysis.

\section{Statistical analysis}

All data are expressed as the mean \pm SE. SPSS 13.0 (SPSS, Inc., Chicago, IL) was used for data analysis. A one- way analysis of variance (ANOVA) or two-tailed Student's $t$ test was used to determine statistical significance between the control and test groups. A one-way ANOVA with Dunnett's posttest for $>2$ groups. A $P$ value of $\leq 0.05$ was considered significant. Analyses were performed using the Graphpad Instat software (San Diego, CA).

\section{Acknowledgements}

This work was supported by the fund from National Nature Science foundation of China (No. 81370818).

\section{Author details}

${ }^{1}$ Department of Nephrology, Union Hospital, Tongji Medical College, Huazhong University of Science and Technology, 430022 Wuhan, China. ${ }^{2}$ Department of Trauma Surgery, Tongji Hospital, Tongji Medical College, Huazhong University of Science and Technology, 430030 Wuhan, China. ${ }^{3}$ Department of Rheumatology, Sun Yat-sen Memorial Hospital, Sun Yat-sen University, 510120 Guangzhou, China. ${ }^{4}$ Department of Medicine, University of California, San Francisco, CA 94107-2140, USA. ${ }^{5}$ Department of Molecular and Cellular Pharmacology, University of California, San Francisco, CA 94107-2140, USA

Conflict of interest

The authors declare that they have no conflict of interest.

\section{Publisher's note}

Springer Nature remains neutral with regard to jurisdictional claims in published maps and institutional affiliations.

Received: 20 June 2018 Revised: 27 September 2018 Accepted: 18 October 2018

Published online: 01 November 2018

\section{References}

1. Nagata, M. Podocyte injury and its consequences. Kidney Int. 89, 1221-1230 (2016).

2. Mundel, P. Podocyte biology and response to injury. J. Am. Soc. Nephrol. 13, 3005-3015 (2002)

3. Pavenstadt, H., Kriz, W. \& Kretzler, M. Cell biology of the glomerular podocyte. Physiol. Rev. 83, 253-307 (2003).

4. Peng, L. et al. Lack of serum- and glucocorticoid-inducible kinase 3 leads to podocyte dysfunction. FASEB J. 32, 576-587 (2018).

5. Li, C., Ge, Y., Dworkin, L., Peng, A. \& Gong, R. The $\beta$ isoform of GSK3 mediates podocyte autonomous injury in proteinuric glomerulopathy. J. Pathol. 239 23-35 (2016).

6. Li, C., Ge, Y., Peng, A. \& Gong, R. The redox sensitive glycogen synthase kinase 3 beta suppresses the self-protective antioxidant response in podocytes upon oxidative glomerular injury. Oncotarget 6, 39493-39506 (2015).

7. Lang, F. et al. (Patho)physiological significance of the serum- and glucocorticoid-inducible kinase isoforms. Physiol. Rev. 86, 1151-1178 (2006).

8. Wang, $X$. et al. Interplay between the Notch and PI3K/Akt pathways in high glucose-induced podocyte apoptosis. Am. J. Physiol. Renal Physiol. 306, F205-F213 (2014).

9. Tejada, T. et al. Failure to phosphorylate AKT in podocytes from mice with early diabetic nephropathy promotes cell death. Kidney Int. 73, 1385-1393 (2008).

10. Canaud, G. et al. AKT2 is essential to maintain podocyte viability and function during chronic kidney disease. Nat. Med. 19, 1288-1296 (2013).

11. Arencibia, J. M., Pastor-Flores, D., Bauer, A. F., Schulze, J. R. O. \& Biondi, R. M. AGC protein kinases: From structural mechanism of regulation to allosteric drug development for the treatment of human diseases. Biochim. Biophys. Acta 1834, 1302-1321 (2013).

12. Kerjaschki, D., Sharkey, D. J. \& Farquhar, M. G. Identification and characterization of podocalyxin-the major sialoprotein of the renal glomerular epithelial cell. J. Cell. Biol. 98, 1591-1596 (1984). 
13. Sawada, H., Stukenbrok, H., Kerjaschki, D. \& Farquhar, M. G. Epithelial polyanion (podocalyxin) is found on the sides but not the soles of the foot processes of the glomerular epithelium. Am. J. Pathol. 125, 309-318 (1986).

14. Doyonnas, R. et al. Anuria, omphalocele, and perinatal lethality in mice lacking the CD34-related protein podocalyxin. J. Exp. Med. 194, 13-27 (2001).

15. Ikenouchi, J., Hirata, M., Yonemura, S. \& Umeda, M. Sphingomyelin clustering is essential for the formation of microvilli. J. Cell. Sci. 126, 3585-3592 (2013).

16. Tesar, V. \& Zima, T. Recent progress in the pathogenesis of nephrotic proteinuria. Crit. Rev. Clin. Lab. Sci. 45, 139-220 (2008).

17. Takeda, T. Podocyte cytoskeleton is connected to the integral membrane protein podocalyxin through $\mathrm{Na}+/ \mathrm{H}+$-exchanger regulatory factor 2 and ezrin. Clin. Exp. Nephrol. 7, 260-269 (2003).

18. Schmieder, S., Nagai, M., Orlando, R. A., Takeda, T. \& Farquhar, M. G. Podocalyxin activates RhoA and induces actin reorganization through NHERF1 and Ezrin in MDCK cells. J. Am. Soc. Nephrol. 15, 2289-2298 (2004).

19. Hara, M. et al. Urinary podocalyxin is an early marker for podocyte injury in patients with diabetes: establishment of a highly sensitive ELISA to detect urinary podocalyxin. Diabetologia 55, 2913-2919 (2012).

20. Asao, R. et al. Relationships between levels of urinary podocalyxin, number of urinary podocytes, and histologic injury in adult patients with IgA nephropathy. Clin. J. Am. Soc. Nephrol. 7, 1385-1393 (2012).

21. Kawaguchi, K., Yoshida, S., Hatano, R. \& Asano, S. Pathophysiological roles of ezrin/radixin/moesin proteins. Biol. Pharm. Bull. 40, 381-390 (2017).

22. Morales, F. C., Takahashi, Y., Kreimann, E. L. \& Georgescu, M. M. Ezrin-radixinmoesin (ERM)-binding phosphoprotein 50 organizes ERM proteins at the apical membrane of polarized epithelia. Proc. Natl Acad. Sci. USA 101, 17705-17710 (2004).

23. Nielsen, J. S. et al. The CD34-related molecule podocalyxin is a potent inducer of microvillus formation. PLOS ONE 2, e237 (2007).

24. Takeda, T., McQuistan, T., Orlando, R. A. \& Farquhar, M. G. Loss of glomerular foot processes is associated with uncoupling of podocalyxin from the actin cytoskeleton. J. Clin. Invest. 108, 289-301 (2001).

25. Lamothe, S. M. \& Zhang, S. Chapter Five - Ubiquitination of ion channels and transporters. Prog. Mol. Biol. Transl. Sci. 141, 161-223 (2016).

26. Foot, N., Henshall, T. \& Kumar, S. Ubiquitination and the regulation of membrane proteins. Physiol. Rev. 97, 253-281 (2017).

27. Yang, B. \& Kumar, S. Nedd4 and Nedd4-2: closely related ubiquitin-protein ligases with distinct physiological functions. Cell Death Differ. 17, 68-77 (2010).

28. Hugo, C. et al. The plasma membrane-actin linking protein, ezrin, is a glomerular epithelial cell marker in glomerulogenesis, in the adult kidney and in glomerular injury. Kidney Int. 54, 1934-1944 (1998).

29. Ostalska-Nowicka, D. Ezrin-a useful factor in the prognosis of nephrotic syndrome in children: an immunohistochemical approach. J. Clin. Pathol. 59, 916-920 (2006).
30. Tao, H. et al. Cyclic AMP prevents decrease of phosphorylated ezrin/radixin/ moesin and chloride intracellular channel 5 expressions in injured podocytes. Clin. Exp. Nephrol. 19, 1000-1006 (2015).

31. $\mathrm{Mo}, \mathrm{H}$. et al. C-X-C chemokine receptor type 4 plays a crucial role in mediating oxidative stress-induced podocyte injury. Antioxid. Redox Signal. 27, 345-362 (2017).

32. Bhalla, V. et al. Serum- and glucocorticoid-regulated kinase 1 regulates ubiquitin ligase neural precursor cell-expressed, developmentally downregulated protein 4-2 by inducing interaction with 14-3-3. Mol. Endocrinol. 19, 3073-3084 (2005).

33. Arroyo, J. P. et al. Nedd4-2 modulates renal Na+-Cl- cotransporter via the aldosterone-SGK1-Nedd4-2 pathway. J. Am. Soc. Nephrol. 22, 1707-1719 (2011).

34. Xu, J., Liu, D., Gill, G. \& Songyang, Z. Regulation of cytokine-independent survival kinase (CISK) by the Phox homology domain and phosphoinositides. J. Cell. Biol. 154, 699-706 (2001).

35. Liu, D., Yang, X. \& Songyang, Z. Identification of CISK, a new member of the SGK kinase family that promotes IL-3-dependent survival. Curr. Biol. 10, 1233-1236 (2000).

36. Fehon, R. G., McClatchey, A. I. \& Bretscher, A. Organizing the cell cortex: the role of ERM proteins. Nat. Rev. Mol. Cell Biol. 11, 276-287 (2010).

37. Wasik, A. A. et al. Ezrin is down-regulated in diabetic kidney glomeruli and regulates actin reorganization and glucose uptake via GLUT1 in cultured podocytes. Am. J. Pathol. 184, 1727-1739 (2014).

38. Bautista-Garc a, P. et al. Zona occludens-2 protects against podocyte dysfunction induced by ADR in mice. Am. J. Physiol. Ren. Physiol. 304, F77-F87 (2013).

39. Zhou, L. et al. Mutual antagonism of Wilms' tumor 1 and beta-catenin dictates podocyte health and disease. J. Am. Soc. Nephrol. 26, 677-691 (2015).

40. Zhou, L. et al. Multiple genes of the renin-angiotensin system are novel targets of Wnt/beta-catenin signaling. J. Am. Soc. Nephrol. 26, 107-120 (2015).

41. Pawluczyk, I. Z. A. et al. Sialic acid attenuates puromycin aminonucleosideinduced desialylation and oxidative stress in human podocytes. Exp. Cell Res. 320, 258-268 (2014).

42. Krishnamurti, $U$. et al. Puromycin aminonucleoside suppresses integrin expression in cultured glomerular epithelial cells. J. Am. Soc. Nephrol. 12, 758-766 (2001).

43. Liu, $X$. et al. Orthogonal ubiquitin transfer identifies ubiquitination substrates under differential control by the two ubiquitin activating enzymes. Nat. Commun. 8, 14286 (2017).

44. Zaarour, R. F. et al. Ezrin ubiquitylation by the E3 ubiquitin ligase, MWP1, and consequent regulation of hepatocyte growth factor receptor activity. PLOS ONE 7, e37490 (2012).

45. Wang, $H$. et al. GIV/girdin links vascular endothelial growth factor signaling to Akt survival signaling in podocytes independent of nephrin. J. Am. Soc. Nephrol. 26, 314-327 (2015). 